\title{
Interactions in processing segmental and suprasegmental features of speech
}

\author{
JOANNE L. MILLER \\ Northeastern University, Boston, Massachusetts 02115
}

\begin{abstract}
A speeded classification task was used to determine the nature of the dependency relation that exists during the processing of a segmental distinction in vowel quality and a suprasegmental distinction in pitch (Experiment 1) or loudness (Experiment 2) in a consonantvowel syllable. In both experiments, evidence was found for a mutual, symmetrical dependency between the processes underlying the analysis of the segmental and suprasegmental information in the syllable. This type of interaction pattern contrasts with the pattern of unidirectional dependency previously found for the analysis of a consonantal distinction and a suprasegmental distinction, in which the analysis of the consonantal information was found to be dependent on the analysis of the suprasegmental information (e.g., Wood, 1974). Together with these earlie: findings, the present results clearly indicate that the form of the interaction between processes responsible for segmental and suprasegmental analysis is a function of the type of segmental information being analyzed. Future research must determine whether the distinct interaction patterns found thus far for consonants and vowels are due to a difference in phonetic class per se, i.e., consonant vs. vowel, or to a difference in the nature of the acoustic information specifying the consonant and vowel.
\end{abstract}

The speech signal simultaneously carries information about the phonetic segments of speech, i.e., the consonants and vowels, and the suprasegmental features of speech, such as the pitch, loudness, and intonation of the utterance. Of importance for theories of speech perception is the manner in which the two types of information are extracted from the acoustic waveform during speech processing.

Although there is evidence to suggest that different processes may be responsible for the analysis of segmental and suprasegmental information (e.g., Blumstein \& Cooper, 1974; Wood, Goff, \& Day, 1971; Sawusch, Pisoni, \& Cutting, Note 1), very little is known about whether these processes operate independently of one another or in an interactive fashion. Wood $(1974,1975)$, however, has recently developed a procedure useful for investigating this issue. He was specifically interested in the perception of two consonants, [b] and [g], which differ from each other only in place of articulation, and in the perception of the suprasegmental feature of pitch. The stimuli in his experiments were four consonant-vowel (CV) syllables, [bae] and [gae], each at two pitch levels. The procedure was a two-choice speeded classifica-

This research was supported by Fellowship NS 00143 from the National Institute of Neurological and Communicative Disorders and Stroke to the author and by Grant HD 05331 from the National Institute of Child Health and Human Development to Peter D. Eimas. I would like to express my gratitude to Dr. Alvin M. Liberman, for his generosity in making available the facilities of the Haskins Laboratories for the preparation of stimulus materials, and to Dr. Peter D. Eimas, for his very helpful comments on an earlier version of this manuscript. tion task that was adapted from the procedures that have been used extensively by Garner and his colleagues to study visual perception (cf. Garner, 1974). In the Wood experiments, subjects classified the syllables either according to pitch (high vs. low) or according to consonant ([b] vs. [g]) under two conditions of variation, a control condition and an orthogonal condition (in the 1974 study a correlated condition, which will be discussed below, was also included). In the control condition, two stimuli were presented that varied along the target dimension, with the value along the nontarget dimension held constant. Thus, in the control condition with pitch as the target dimension, subjects classified either the two [bae]s or the two [gae]s, and in the control condition with the consonantal distinction as the target dimension, they classified either the two syllables with high pitch or the two syllables with low pitch. In the orthogonal condition, all four stimuli were presented, with the subjects again classifying the syllables according to the consonantal or pitch distinction. Of interest was the comparison of decision times in the control and orthogonal conditions for each target feature. The extent to which random variation along the nontarget dimension in the orthogonal condition slows reaction time over that obtained in the control condition is assumed to reflect the degree to which analysis of the target feature is dependent on the analysis of the nontarget feature (cf. Eimas, Tartter, Miller, \& Keuthen, 1978; Wood, 1975).

In both studies, Wood found that the interference caused by orthogonal variation in the nontarget dimension was asymmetric: When the subject was 
targeting for pitch, there was no reliable increase in classification time in the orthogonal condition; however, when the target dimension was the consonant, variation in pitch interfered with processing, resulting in reliably higher response times in the orthogonal than in the control condition. Goldin (Note 2) has recently extended these findings by showing that this form of asymmetry is not limited to the particular dimensions of place of articulation and pitch that were used by Wood. She obtained asymmetric interference when the two dimensions were place and loudness and when they were voicing (e.g., [b] vs. [p]) and pitch.

Taken together, the results of the Wood and Goldin studies suggest that there is a unidirectional dependency in the processing of segmental and suprasegmental information, with the analysis of segmental information dependent on the prior analysis of suprasegmental information. The analysis of suprasegmental features, on the other hand, is accomplished independently of segmental analysis. However, in each of these studies, despite the fact that the stimuli were CV syllables, the segmental distinction always involved the consonant; that is to say, the vowel quality of the syllable remained unchanged. The purpose of the present research was to determine whether a pattern of asymmetric interference would also be obtained when a contrast in vowel quality, rather than in consonantal identity, was paired with a contrast in a suprasegmental feature. If the critical factor governing the type of interaction is simply whether the analysis involves segmental and suprasegmental information, then of course vowels should exhibit the same pattern with respect to suprasegmental features as do consonants. However, there is a considerable body of evidence, including studies of dichotic listening (e.g., Studdert-Kennedy \& Shankweiler, 1970), categorical perception (e.g., Eimas, 1963; Pisoni, 1973), and anchoring effects (e.g., Simon, 1977), that has shown that under certain conditions consonants and vowels, especially in simple CV syllables of the type used by Wood (1974, 1975) and Goldin (Note 2), may undergo different forms of processing. Therefore, we might expect the form of interaction between segmental and suprasegmental processing to differ when the segmental distinction involves the vowel, rather than the consonant, of the syllable.

To investigate this possibility, two experiments were conducted using the speeded classification task. This procedure was considered to be particularly well suited for the investigation of dependencies in that it had proved capable of revealing different, but consistent, dependency patterns with various aspects of speech. For example, Wood (1975) found evidence for a mutual dependency in the processing of two suprasegmental features of a syllable, either pitch and loudness or pitch and pitch contour and, similarly, Wood and Day (1975) reported evidence for a mutual dependency in the processing of two segmental dimensions, the consonant and vowel of a CV syllable. Finally, in a series of studies, Eimas et al. (1978) found the processing of place and manner of articulation to show a mutual, asymmetric dependency, with the analysis of place information more dependent on the analysis of manner information than the reverse.

The stimuli for each of the two current experiments were four CV syllables, [ba] and [bae], each at two levels of pitch (Experiment 1), and [ba] and [bae], each at two loudness levels (Experiment 2). Within each experiment, the syllables were classified under three conditions of variation, a control and an orthogonal condition, as described above, and a correlated condition. In the correlated condition, two stimuli were presented that differed along both dimensions, for example, a high [ba] and a low [bae]. Both Wood (1974) and Goldin (Note 2) also included such a correlated condition in their experiments; however, they obtained different results. Wood found a mutual redundancy gain, such that decision times for both targets (consonant and pitch) were faster in the correlated than in the control condition. Wood interpreted that finding as indicating that although listeners could ignore variation in consonantal quality while making pitch judgments (as indicated by the unidirectional interference effect in the orthogonal condition), they could use redundant variation in consonantal quality and pitch to speed pitch judgments. Goldin, however, failed to find any evidence of a true redundancy effect in either of her studies. In accord with these findings, Eimas et al. (1978) also did not find a true redundancy gain in their studies on place and manner of articulation. ${ }^{1}$ Although the correlated condition has yielded somewhat equivocal results with speech stimuli, and despite the fact that it is not essential for conclusions about the nature of processing, it was included in the present experiments for the sake of completeness.

\section{METHOD}

\section{Subjects}

The subjects in each experiment were 12 Brown University students, who were paid for their participation. ${ }^{2}$ Two of the subjects who participated in the first study also served as listeners in the second study. None of the subjects reported any history of a speech or hearing disorder.

\section{Stimuli}

The stimuli for Experiment 1 were four three-formant $\mathrm{CV}$ syllables, [ba] and [bae], each at a high and low pitch, that were generated on the Haskins Laboratories parallel resonance synthesizer. Each syllable was $265 \mathrm{msec}$ in duration, including an initial formant transition period of $45 \mathrm{msec}$. All stimuli had a falling amplitude contour and the fundamental frequency remained constant at $104 \mathrm{~Hz}$ for the two syllables with low pitch and at $140 \mathrm{~Hz}$ 
for the two syllables with high pitch. For [ba], the starting and steady-state formant frequency values were, respectively, 181 and $769 \mathrm{~Hz}(\mathrm{~F} 1), 921$ and $1,232 \mathrm{~Hz}(\mathrm{~F} 2)$, and 2,018 and $2,525 \mathrm{~Hz}$ (F3); for [bae], these values were 181 and $743 \mathrm{~Hz}(\mathrm{~F} 1), 1,312$ and $1,620 \mathrm{~Hz}(\mathrm{~F} 2)$, and 2,348 and $2,862 \mathrm{~Hz}(\mathrm{~F} 3)$, For Experiment 2, the stimuli were also four $\mathrm{CV}$ syllables, in this case [ba] and [bae] each at two loudness levels. The two loud syllables were the two syllables with low pitch used in Experiment 1; the two soft syllables were generated by attenuating each of these stimuli by $20 \mathrm{~dB}$.

For each experiment, eight stimulus tapes were constructed using the PCM system at the Haskins Laboratories, including four control tapes, two correlated tapes, and two orthogonal tapes. Each control tape contained instances of two stimuli that differed along one dimension. Thus, for example, in Experiment 1 there were two pitch control tapes, one with [ba] at both pitch levels and one with [bae] at both pitch levels, and two vowel control tapes, one with the low [ba] and [bae] and one with the high [ba] and [bae]. The two correlated tapes for each experiment contained instances of two stimuli that differed along both dimensions. Thus in Experiment 1, for example, one tape contained the high [ba] and the low [bae] and the other contained the low [ba] and the high [bae]. Each of the orthogonal tapes contained instances of all four stimuli used in that experiment. On each tape there were 12 practice items followed by 64 randomized test items, for a total of 76 stimuli per tape, and on a given tape each stimulus occurred equally often. All syllables were recorded on one channel of the audio tape with a synchronized signal, which activated a timer, recorded on the other channel. The stimuli were recorded with an interstimulus interval of $4 \mathrm{sec}$.

\section{Procedure}

The procedure was identical in the two experiments. Each subject was run individually in one experimental session in which he classified the syllables according to each dimension, vowel and pitch (Experiment 1) or vowel and loudness (Experiment 2), under each of three conditions of variation, correlated, control, and orthogonal. Thus each subject judged six of the test tapes in all. The particular control tape classified for each dimension and which correlated tape was classified according to which dimension were counterbalanced across subjects. The order of the six tests (two dimensions by three conditions) was determined by a balanced Latin square, with the property that each test precede and follow every other test once. The various stimulus sets used for each condition are presented in Tables 1 and 2 for Experiments 1 and 2 , respectively.

At the beginning of the session, the subject was given a general explanation of the procedures to be followed and before each of the six tests he was told which syllables he would hear on that block of trials and which dimension to use for classification. The subject was further instructed to place the index finger of his preferred hand on a neutral center button and to indicate his response by pressing the appropriately labeled response button located to the right or left of center. The labels, A and AE for the vowel distinction and High and Low for the pitch distinction (Experiment 1) and $\mathrm{A}$ and $\mathrm{AE}$ for the vowel distinction and Loud and Soft for loudness distinction (Experiment 2), were counterbalanced for position across subjects. The subjects were encouraged to respond as quickly as possible on each trial without sacrificing accuracy for speed.

The stimulus tapes were played on a Crown SX-822 tape recorder and presented to the listener through Koss Pro 600AA earphones. In Experiment 1, all syllables were presented at $78 \mathrm{~dB}$ SPL, and in Experiment 2, the loud syllables were again presented at $78 \mathrm{~dB} \mathrm{SPL}$ and the soft syllables were presented at $58 \mathrm{~dB}$ SPL. The signal on the second channel of the tape was shunted to a voice-activated relay (Lafayette Model 1604A), which in turn activated a digital timer accurate to $1 \mathrm{msec}$ (Hunter Model 1521). The timer was stopped by the subject's buttonpress.

Table 1

Stimulus Sets Used for Each Target Dimension in Each Condition (Experiment 1)

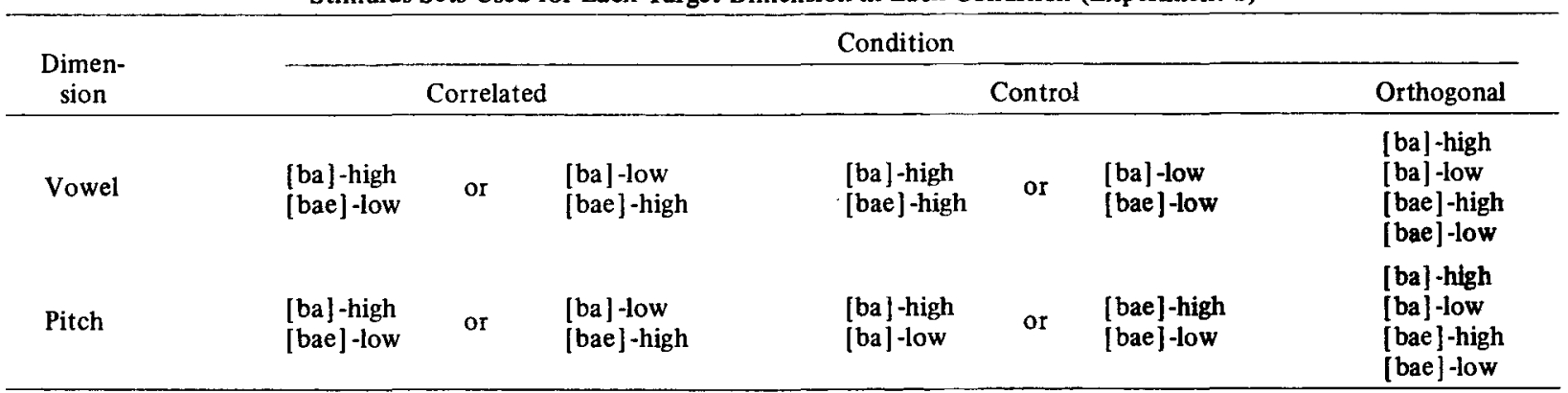

Table 2

Stimulus Sets Used for Each Target Dimension in Each Condition (Experiment 2)

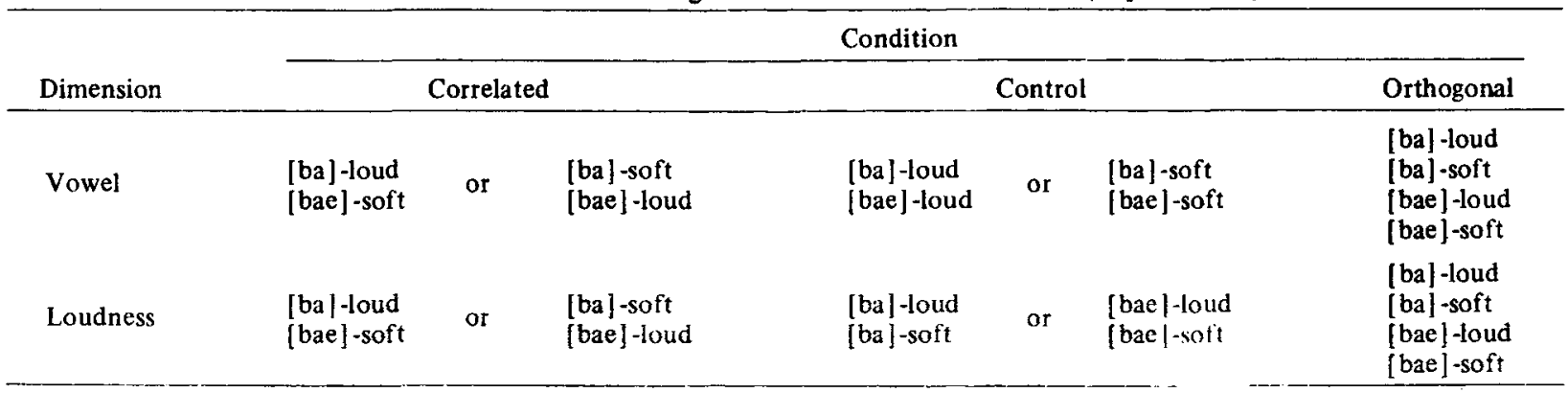




\section{RESULTS}

\section{Experiment 1}

Subjects performed the task quite accurately, responding with an average overall error rate of less than $2 \%$. Moreover, a Friedman analysis of variance indicated that errors were randomly distributed across conditions.

Of primary interest, then, are the reaction time data for correct responses. Mean reaction times for the six tests are presented in Table 3. A preliminary analysis (Friedman) indicated that there was no effect of order of condition on the decision time scores. The individual mean scores were entered into a repeated measures analysis of variance, Condition by Target by Subject, which yielded one reliable effect, that of Condition $[F(2,22)=24.18, p<.001]$. Individual comparisons revealed that this was due to longer times in the orthogonal than in the control or correlated conditions, with the control and correlated means not reliably different. Thus there was an interference effect but no redundancy effect. The lack of a significant Target by Condition interaction indicated further that the increase in reaction time in the orthogonal condition did not differ reliably as a function of target. These results indicate that, unlike the asymmetric interference effect for consonant and pitch, the interference effect for vowel and pitch is both mutual and symmetrical.

\section{Experiment 2}

The pattern of results in this experiment was essentially the same as that of the first experiment. First, subjects again made very few errors (less than $1 \%$ overall) and those that were made were randomly distributed across conditions (Friedman analysis of variance). The mean reaction times for correct responses for the six tests are given in Table 4. As in the first study, a preliminary analysis (Friedman) indicated no effect of order of conditions on the decision times. An analysis of variance, Condition by Target by Subject, performed on the individual mean reaction time scores, revealed two significant effects, one due to Target $[\mathrm{F}(1,11)=15.88, \mathrm{p}<.005]$ and one due to Condition $[F(2,22)=6.37, p<.01]$. The target effect was due to lower decision times when the target dimension was loudness than when it was

Table 3

Mean Reaction Time in Milliseconds for Vowel and Pitch Target Dimensions in Each Experimental Condition (Experiment 1)

\begin{tabular}{llcc}
\hline & \multicolumn{3}{c}{ Condition } \\
\cline { 2 - 4 } $\begin{array}{c}\text { Dimen- } \\
\text { sion }\end{array}$ & $\begin{array}{c}\text { Corre- } \\
\text { lated }\end{array}$ & Control & $\begin{array}{c}\text { Ortho- } \\
\text { gonal }\end{array}$ \\
\hline Vowel & 540 & 561 & 603 \\
Pitch & 542 & 558 & 628 \\
\hline
\end{tabular}

Table 4

Mean Reaction Time in Milliseconds for Vowel and Loudness Target Dimensions in Each Experimental Condition (Experiment 2)

\begin{tabular}{lccc}
\hline \multirow{2}{*}{$\begin{array}{c}\text { Dimen- } \\
\text { sion }\end{array}$} & $\begin{array}{c}\text { Corre } \\
\text { lated }\end{array}$ & Control & $\begin{array}{c}\text { Ortho- } \\
\text { gonal }\end{array}$ \\
\hline Vowel & 460 & 466 & 501 \\
Loudness & 438 & 443 & 472 \\
\hline
\end{tabular}

vowel. The lack of an interaction of Target with Condition indicated, however, that this difference in reaction time was constant across conditions. Individual comparisons revealed that the Condition effect was due to increased reaction times in the orthogonal condition as compared to the control and correlated conditions, with no reliable difference between control and correlated times. Thus, as in the first study, there was an interference effect but no redundancy effect. Finally, the lack of a Condition by Target interaction signified that again the magnitude of the interference effect did not reliably differ as a function of the target dimension.

\section{DISCUSSION}

The two experiments reported in this paper yielded an identical pattern of results. When subjects were asked to make a judgment regarding the vowel of a syllable and a judgment regarding either its pitch or loudness, redundant variation in the two dimensions had no reliable effect on classification times, whereas orthogonal variation significantly slowed decision times along both dimensions.

The failure to find a redundancy effect in either study is consistent with the results of both Eimas et al. (1978) and Goldin (Note 2) although it is discrepant from those of Wood (1974). Thus, with the exception of Wood's data, the available evidence indicates that when speed stimuli are used, no redundancy gain is obtained. ${ }^{3}$ Inasmuch as the lack of a redundancy gain seems to occur regardless of the type of interference pattern obtained, whether unidirectional (Goldin), mutual but asymmetric (Eimas et al.), or mutual and symmetrical (the present two studies), it may be that the correlated condition is simply not appropriate for revealing differences in processing patterns that are, in fact, revealed by the orthogonal condition.

Of primary importance for the present argument, then, is the pattern of results found in the control and orthogonal conditions. In both studies, there was a mutual, symmetrical interference effect, with orthogonal variation slowing reaction times for both target dimensions. Before considering the implications of this finding for speech processing, however, 
it is necessary to eliminate the possibility that this pattern was simply due to an artifact of stimulusresponse mapping (cf. Hick, 1952). Recall that in the control condition only two stimuli were presented, one to be mapped to each response, but that in the orthogonal condition four stimuli were presented, two to be mapped to each response. Thus, the increase in reaction times in the orthogonal condition may have been due to the increased complexity of the task and not due to factors concerning speech processing, per se. However, it is very unlikely that this is the case. Although in both of the current studies there was an increase in the orthogonal condition for both target dimensions, this procedure does not always yield such a result. For example, as discussed above, using identical procedures, both Wood (1974, 1975) and Goldin (Note 2) found an increase in orthogonal reaction times over control times only for one dimension. In addition, the same speeded classification task has been used in a number of studies on the processing of nonspeech auditory stimuli in which unidirectional interference patterns were also obtained (Blechner, Day, \& Cutting, 1976; Pastore, Ahroon, Puleo, Crimmins, Golowner, \& Berger, 1976). A simple stimulus-response mapping account cannot explain why an increase in reaction time should occur only for one dimension. Inasmuch as this asymmetric pattern is often obtained, the fact that an increase occurred for both dimensions in the current studies most likely reflects true processing interactions and not simply stimulus-response mapping factors.

The mutual, symmetrical interference effects found in the present studies, together with the asymmetric interference effects reported by Wood and Goldin, clearly indicate that there is not a single type of interaction in the processing of segmental and suprasegmental information. While the analysis of the consonantal information and pitch (or loudness) was found to be done in a hierarchical or serial fashion, with consonantal analysis dependent on analysis of the suprasegmental information, the analyses required to determine vowel quality and pitch (or loudness) were found to operate in a mutually dependent fashion.

A critical issue to be addressed by future research is whether these differences in processing interactions were due to a difference in phonetic class per se, consonant vs. vowel, or to differences in acoustic structure. That is, in the current studies and in those of Wood and Goldin, the consonant was primarily cued by rapidly changing formant frequency information, whereas the vowel was primarily cued by longer, steady-state information. Research using other paradigms has shown that the way in which the consonant and vowel are specified is important, and that differences in processing patterns typically found for consonants and vowels can be minimized or eliminated by changing the type of acoustic information specifying each (e.g., Darwin, 1971; Darwin \& Baddeley, 1974; Fujisaki \& Kawashima, 1969; Stevens, 1968). Thus, it may be that manipulations in the way in which the consonant and vowel are cued will also change the way in which processing of the segmental information interacts with processing of the suprasegmental information. Of particular importance will be to determine whether a pattern of mutual, symmetrical dependency between processes underlying vowel and suprasegmental analysis, found with simple CV syllables, will also be found using syllables that more closely approximate natural speech, in which both the vowel and consonant are largely specified by the formant transitions (cf. Shankweiler, Strange, \& Verbrugge, 1977). However, such manipulations in acoustic structure may not change the distinct interaction patterns found thus far for consonants and vowels. In their studies on place and manner of articulation, Eimas et al. (1978) found the identical dependency pattern (mutual, asymmetric dependency) with a variety of particular place and manner combinations cued in a number of ways. Thus this paradigm may be tapping a level of processing that is not sensitive to differences in acoustic characteristics and, if so, the difference in processing found for the consonant and vowel in a CV syllable may obtain regardless of the way the segmental value is cued.

In summary, then, the present experiments clearly indicate that first, during the perception of speech, there is an interaction between the processes responsible for analysis of the segmental and suprasegmental information in the waveform and, second, the type of interaction varies with changes in the segmental information being analyzed. Whether such variation in interaction pattern arises because of a difference in the phonetic class of the segmental information (consonant vs. vowel) or a difference in the nature of the acoustic information specifying the phonetic segment remains to be determined.

\section{REFERENCE NOTES}

1. Sawusch, J. R., Pisoni, D. B., \& Cutting, J. E. Category boundaries for linguistic and nonlinguistic dimensions of the same stimuli. Paper presented at the 87th meeting of the Acoustical Society of America, April, 1974, New York.

2. Goldin, S. E. Unpublished studies. Brown University, 1975.

\section{REFERENCES}

Blechner, M. J., Day, R. S., \& Cutring, J. E. Processing two dimensions of nonspeech stimuli: The auditory-phonetic distinction reconsidered. Joumal of Experimental Psychology: Human Perception and Performance, 1976, 2. 257-260.

Bl.umstein. S. E.. \& CoOper. W. E. Hemispheric processing of intonation contours. Cone'x. 1974. 10. 146-158.

DARWIN. C.J. Fat differences in the recall of fricatives and 
vowels. Quarterly Journal of Experimental Psychology, 1971. 23, 46-62.

Darwin, C. J., \& Baddeley, A. D. Acoustic memory and the perception of speech. Cognitive Psychology, 1974, 6, 41-60.

Eimas, P. D. The relation between identification and discrimination along speech and nonspeech continua. Language and Speech, 1963, 6, 206-217.

Eimas, P. D., Tartter, V. C., Miller, J. L., \& Keuthen, N. J. Asymmetric dependencies in processing phonetic features. Perception \& Psychophysics, 1978, 23, 12-20.

Fuisaki, H., \& Kawashima, T. On the modes and mechanisms of speech perception. Annual Report of the Engineering Research Institute, Faculty of Tokyo, 1969, 28, 67-73.

GARNER, W. R. The processing of information and structure. Potomac, Md: Erlbaum, 1974.

Hick, W. E. On the rate of gain of information. Quarterly Journal of Experimental Psychology, 1952, 4, 11-26.

Pastore, R. E., Ahroon, W. A., Puleo, J. S., Crimmins, D. B., Golowner, L., \& Berger, R. S. Processing interaction between two dimensions of nonphonetic auditory signals. Journal of Experimental Psychology: Human Perception and Performance, 1976, 2, 267-276.

Prsoni, D. B. Auditory and phonetic memory codes in the discrimination of consonants and vowels. Perception \& Psychophysics, 1973, 13, 253-260.

Shan KWeiler, D., Strange, W., \& Verbrugge, R. Speech and the problem of perceptual constancy. In R. Shaw \& J. Bransford (Eds.), Perceiving, acting and knowing. Hillsdale, N.J: Erlbaum, 1977.

Simon, H. Anchoring and selective adaptation of phonetic and nonphonetic categories in speech perception. Unpublished $\mathrm{PhD}$ thesis, City University of New York, 1977.

STEvens, K. N. On the relations between speech movements and speech perception. Zeitschrift fur Phonetik, Sprachwissenschaft und Kommunikationsforschung, 1968, 21, 102-106.

Studdert-Kennedy, M., \& Shankweiler, D. Hemispheric specialization for speech perception. Joumal of the Acoustical Society of America, 1970, 48, 579-594.

Wood, C. C. Parallel processing of auditory and phonetic information in speech discrimination. Perception \& Psychophysics, 1974, 15, 501-508.

WOOD, C. C. Auditory and phonetic levels of processing in speech perception: Neurophysiological and informationprocessing analyses. Journal of Experimental Psychology: Human Perception and Performance, 1975, 1, 3-20.

Wood, C. C., \& DAY, R. S. Failure of selective attention to phonetic segments in consonant-vowel syllables. Perception \& Psychophysics, 1975, 17, 346-350.

Wood, C. C.. GoFf. W. P., \& DAY, R. S. Auditory evoked potentials during speech perception. Science, 1971, 173, 1248-1251.

\section{NOTES}

1. In certain instances in the Eimas et al. (1978) and Goldin (Note 2) studies, a correlated mean for a given target was actually reliably less than its respective control mean. However, before this can be interpreted as a true correlated effect, with redundant variation between two dimensions speeding processing, it is necessary to rule out the possibility that the mean reduction was due to a selective serial processing (SSP) strategy on the part of the subjects. In using a strategy of SSP, a given subject classifies the stimuli in the correlated condition according to his faster control dimension. Since in no case in which a reliable mean reduction in the correlated condition was obtained was it, in fact, possible to rule out such a strategy, there is no evidence for a true redundancy gain in these studies.

2. One subject was dropped from the first experiment because of exceeding difficulty with the task, resulting both in very high error rates and slow reaction times.

3. It is not clear why Wood (1974) did in fact obtain a significant redundancy effect for speech, although Eimas et al. (1978) argue that the reported effect may have been due to the inappropriate use of statistics. For a further discussion of this issue the reader is referred to Eimas et al.

(Received for publication October 6, 1977; revision accepted April 13, 1978.) 